\title{
High grade foamy gland prostatic adenocarcinoma, a rare pathological variant treated with radical prostatectomy
}

\author{
Malhasi Gunawardena ${ }^{1}$, Umesh Jayarajah ${ }^{2}$, Susantha de Silva ${ }^{2}$, Serozsha Goonewardena ${ }^{2}$, Chandu de Silva ${ }^{1}$ \\ ${ }^{1}$ Department of Pathology, Faculty of Medicine, University of Colombo, Colombo, Sri Lanka \\ ${ }^{2}$ Department of Urology, National Hospital of Sri Lanka, Colombo, Sri Lanka
}

Keywords: : Prostatic adenocarcinoma; small acinar adenocarcinoma; foamy gland carcinoma; Gleason score; International Society of Urological Pathology grade; prostatic specific antigen; estrogen receptors

\section{Introduction}

Prostatic adenocarcinoma is one of the commonest internal malignancies among men and its incidence is increasing with age [1]. Foamy gland carcinoma is one of the variants usually seen in combination with small acinar or usual adenocarcinoma and the pure form is rare $(0.2-2 \%)[1,2]$. It is usually assigned as a low-grade tumour; the most common Gleason score being 7, although some authors have described it as $6[1,2]$. We describe a rare high- grade, pure foamy gland adenocarcinoma occurring in a relatively young patient.

\section{Case Presentation}

A 49-year old male presented with acute urinary retention. He had a preceding history of poor flow of urine for 1-year duration associated with frequency, nocturia and nocturnal enuresis. He was catheterised by the general surgical team and was referred to the urology unit for further care. Digital rectal examination revealed a clinically malignant, hard irregular prostate with obliterated medium groove. His initial serum creatinine was $254 \mu \mathrm{mol} / \mathrm{L}$ which gradually declined to $124 \mu \mathrm{mol} / \mathrm{L}$ after catheterization. His other basic biochemistry and complete blood count were unremarkable. Ultrasonography showed normal kidneys without any hydronephrosis or hydroureter. The bladder wall thickness was $6 \mathrm{~mm}$. The prostate size was $39 \mathrm{~cm} 3$. The prostatespecific antigen level was $1.5 \mathrm{ng} / \mathrm{ml}$.

Cystoscopic examination showed enlarged lateral prostatic lobes which were almost meeting in the midline. The bladder was trabeculated with a high neck. He underwent transurethral resection of the prostate. Microscopic examination revealed prostatic tissue containing a small acinar type prostatic carcinoma predominantly composed of poorly formed and fused nests of tumour cells with foamy

Correspondence: Chandu de Silva

E-mail: desilva vipula@yahoo.com

(iDhttps://orcid.org/0000-0003-2190-6672

Received: 13-08-2020 Accepted: 29-08-2020 DOI: http://doi.org/10.4038/sljs.v38i2.8702

The Sri Lanka Journal of Surgery 2020; 38(2): 80-82

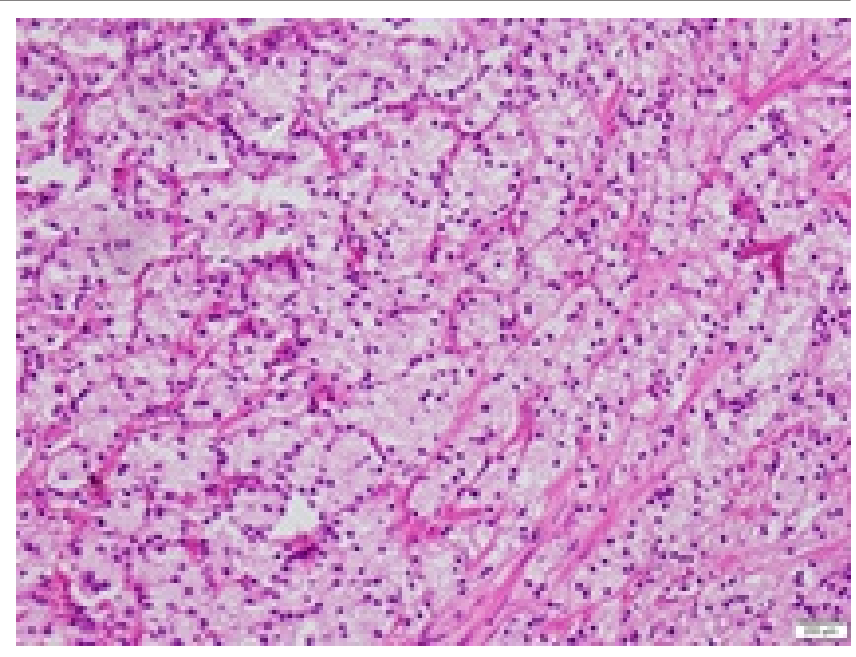

Figure 1. $\mathrm{H}$ and $\mathrm{E}$ stain with $100 \mathrm{X}$ magnifications showing prostatic tissue containing a small acinar type prostatic carcinoma predominantly composed of poorly formed and fused nests of tumour cells with foamy cytoplasm with enlarged nuclei and prominent nucleoli (Gleason score 5).

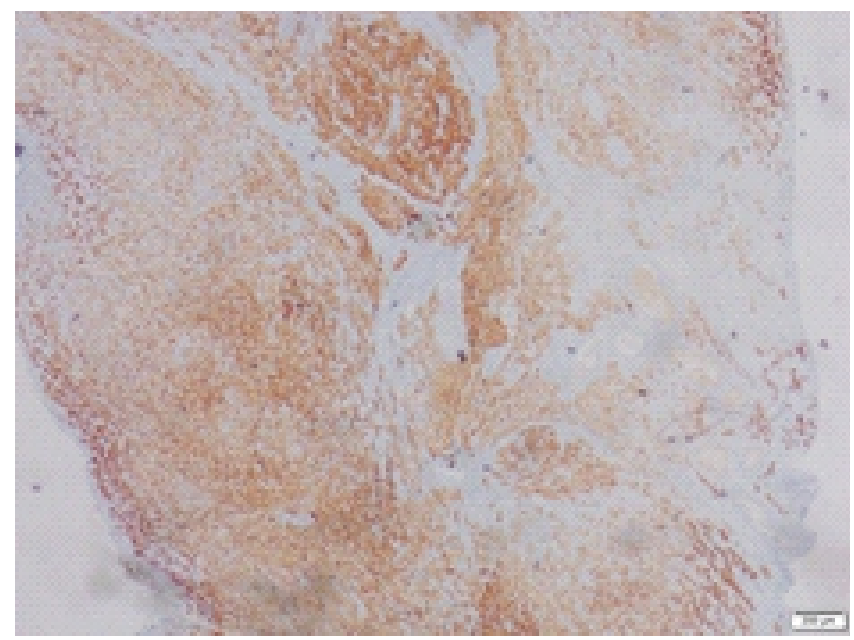

Figure 2. $\mathrm{H}$ and $\mathrm{E}$ stain with $40 \mathrm{X}$ magnifications showing diffuse, moderate-strong cytoplasmic positivity for PSA

cytoplasm(Gleason score 5)(figure 1). The nuclei were enlarged and round with prominent nucleoli. Necrosis was not seen. There was focal perineural invasion (figure S1). Lymphovascular invasion and prostatic intraepithelial neoplasia were absent. Thus the histological findings were in 
favour of high-grade foamy gland (pure) prostatic adenocarcinoma- with more than $80 \%$ tumour burden. The Gleason score was $5+4$ and International Society of Urological Pathology group(ISUP) grade was 5 .

The tumour cells showed diffuse, moderate-strong cytoplasmic positivity for Prostate-specific antigen (PSA) immunostaining(figure 2). Estrogen receptor (ER) immunostaining was negative in tumour cells, however, some stromal cells showed positive nuclear staining.

Computed tomography of the abdomen and magnetic resonance imaging (MRI) of the pelvis was performed and there was no evidence of local invasion or distant metastasis. Therefore, following a multidisciplinary team discussion a radical prostatectomy was performed. Exploration revealed bilateral pelvic lymphadenopathy and hard prostate. Therefore, bilateral lymphadenectomy was performed with radical prostatectomy.

Histology revealed a tumour burden of $47 \%$. Gleason sum score was $5+4$ and ISUP grade was 5 . Established extraprostatic extension with circumferential involvement of anterior surface close to bladder neck with seminal vesicle invasion was seen (figure 2). There was no lymphovascular invasion or high grade prostatic intraepithelial neoplasia. Four of nine right pelvic nodes contained metastatic deposits and all four left pelvic lymph nodes were negative. Pathological stage was pT3bN1.

He underwent hormonal therapy with a course of Goserelin and Bicalutamide. He was asymptomatic without any evidence of recurrence at 12 months after surgery.

\section{Discussion}

Foamy gland carcinoma is characterized by abundant foamy cytoplasm and often pyknotic nuclei $[1,2]$. The nucleoli are usually inconspicuous and the cytological atypia is minimal $[1,2]$.

Zhao et al have reported a series of 55 patients with similar histological morphology, in which enlarged nuclei and prominent nucleoli were reported in approximately $80 \%$ and $70 \%$, respectively [2]. The commonest morphological patterns were cribriform (73\%) and poorly defined (fused) glands $(55 \%)$. Less common patterns were cords single cells/ cords and solid sheets [2].

Certain variants of high-grade foamy gland adenocarcinoma of the prostate were found to be difficult to diagnose as scanty amounts of bland foamy glands were seen scattered within a dense sclerosed desmoplastic stroma [2]. Diagnostic awareness of the existence of this variant is important for accurate diagnosis of prostatic cancer [1,3]. The clinical behaviour of this variant is usually aggressive despite the benign histologic appearance, although Hudson et al. has reported 100 cases of foamy gland adenocarcinoma without a significant difference in prognosis compared with usual acinar adenocarcinoma [3].

Koca et al have studied 56 cases of foamy gland adenocarcinoma of the prostate based on core needle prostate biopsies. They found that these foamy gland adenocarcinomas had similar features to conventional prostatic adenocarcinoma (acinar type) in relation to clinical features such as age, biochemical features such as prostatespecific antigen titres and histological characteristics such as the presence of high grade prostatic intraepithelial neoplasia and Gleason's score [4]. The prostate-specific antigen serves as a useful immunohistochemical marker for prostatic epithelium with a great degree of specificity and sensitivity $[1$, 2]. It is useful in confirming a difficult diagnosis such as foamy gland carcinoma, although it does not distinguish benign and malignant processes in prostate [1].

It is demonstrated that estrogens are required for prostate development and carcinogenesis [5]. The ER- $\beta$ immunostaining gives a strong nuclear positivity in low-grade prostatic adenocarcinoma and it was negative in some highgrade carcinomas [5]. ER- $\alpha$ expression in stroma could play protective roles to reduce prostatic cancer development by decreasing androgen production [5]. Selective estrogen receptor modulators may potentially be used in combination with current therapies in the treatment of prostatic carcinoma [5].

It is important to highlight that in the presence of a normal serum PSA value $(<4 \mathrm{ng} / \mathrm{mL})$ only a digital rectal examination by an experienced finger could clinch an accurate clinical diagnosis. A clinically benign prostate on digital rectal examination, in this case, would have led to pharmacological treatment precluding a tissue diagnosis.

\section{Conclusion}

Awareness of the occurrence of rare high-grade type foamy gland carcinoma will help to prevent misdiagnosis of this entity as a non-prostatic malignancy, such as urothelial carcinoma or metastatic deposits, especially when PSA is low. Furthermore, it is important to highlight that in the presence of a normal serum PSA value, only a digital rectal examination by an experienced finger could clinch an accurate clinical diagnosis. Stromal ER could play a protective role to reduce invasion of prostatic carcinoma and selective ER modulators may be used to treat prostatic carcinoma. 
All authors disclose no conflict of interest. The study was conducted in accordance with the ethical standards of the relevant institutional or national ethics committee and the Helsinki Declaration of 1975, as revised in 2000 .

\section{References}

1. Humphrey PA, Moch H, Cubilla AL, Ulbright TM, Reuter VE: The 2016 WHO classification of tumours of the urinary system and male genital organs - part B: prostate and bladder tumours. European urology 2016, 70(1):106-119.

https://doi.org/10.1016/j.eururo.2016.02.028

2. Zhao J, Epstein JI: High-grade foamy gland prostatic adenocarcinoma on biopsy or transurethral resection: a morphologic study of 55 cases. The American journal of surgical pathology 2009, 33(4):583-590

https://doi.org/10.1097/pas.0b013e31818a5c6c

3. Hudson J, Cao D, Vollmer R, Kibel AS, Grewal S, Humphrey PA: Foamy gland adenocarcinoma of the prostate: incidence, Gleason grade, and early clinical outcome. Human pathology 2012, 43(7):974-979. https://doi.org/10.1016/j.humpath.2011.09.009
4. Koca SB, Yıldız P, Behzatoğlu K: Foamy gland carcinoma in core needle biopsies of the prostate: clinicopathologic and immunohistochemical study of 56 cases. Annals of diagnostic pathology 2014, 18(5):271-274.

https://doi.org/10.1016/j.anndiagpath.2014.07.002

5. Yeh C-R, Da J, Song W, Fazili A, Yeh S: Estrogen receptors in prostate development and cancer. American journal of clinical and experimental urology 2014, 2(2):161.

https://europepmc.org/article/pmc/pmc4219301

\section{Abbreviations}

$\begin{array}{ll}\text { ISUP } & \text { : International Society of Urological Pathology } \\ \text { PSA } & \text { : Prostate specific antigen } \\ \text { ER } & \text { : Estrogen receptor } \\ \text { MRI } & \text { : Magnetic resonance imagine }\end{array}$

\section{Learning Points:}

- High-grade, pure foamy gland adenocarcinoma can rarely occur in young patients

- Clinicians should be aware of this rare variety to prevent misdiagnosis of this entity as a non-prostatic malignancy

- Digital rectal examination by an experienced finger is needed to clinch an accurate clinical diagnosis in the absence of elevated PSA levels. 